\title{
Knowledge and Prescription Habits Toward Preoperative Antibiotics in Implant Dentistry: A Survey Analysis in a Subset of Dentists in Saudi Arabia
}

\author{
Mohammed A AlSarhan
}

\begin{abstract}
Aim: To investigate and evaluate current knowledge and habits in prescribing preoperative antibiotic, and toward specific practice situations, in a subset of dental practitioners performing routine dental implant surgery in Saudi Arabia.

Materials and methods: A cross-sectional questionnaire-based study was developed, and relevant information was gathered through a web-based survey from a dentist who performed surgical implant placement. Participant demographics, clinical experience, and educational background were obtained. Factors related to knowledge and practice of preoperative antibiotics prescription and relevant information were collected. Descriptive statistics (frequencies and percentages) were used to describe the categorical variables. Pearson's Chi-square test was used to compare the distribution of the categorical responses across specific survey variables. A $p$ value of $\leq 0.05$ was used to report the statistical significance of the responses relative to prescription habits.

Results: A total of 156 dentists participated in this study. Majority of the study sample was periodontists $(70.5 \%, n=110)$. About $63.5 \%$ of the study subjects do not prescribe prophylactic antibiotics. Private practitioners prescribed more antibiotics preoperatively $(p=0.019)$, while public and academic practitioners were in favor of developing guidelines toward antibiotics prescriptions $(p=0.009)$. Furthermore, a statistically significant difference was found between private and no private practitioners toward possible adverse complications when prescribing multidose antibiotics $(p=0.014)$.

Conclusion: Various factors influence the knowledge and prescription habits toward prophylactic antibiotics among dental practitioners in routine dental implant procedures. The variability and conflicting practices require the attention of health-care legislations and stakeholders, locally and globally, to improve antibiotics prescription habits. Furthermore, large-scale interventions, prescription stewardship programs, and collaborative work between professional and scientific organizations may be beneficial to address areas of concern.

Clinical significance: Strategic policies and stewardship programs toward antibiotic prescription among dental practitioners may benefit in reducing the unjustified or misuse of these medications. Subsequently, this could minimize the potential development of bacterial resistance and unwanted events that might complicate the management of straightforward dental implant cases.

Keywords: Antibiotics, Habits, Implants, Preoperative, Saudi Arabia.

The Journal of Contemporary Dental Practice (2020): 10.5005/jp-journals-10024-2798
\end{abstract}

\section{INTRODUCTION}

Dental implants are considered one of the major game changers in dentistry. ${ }^{1}$ The popularity of using dental implants among dental practitioners and the increase demand of patients to have fixed tooth replacement is evident in published literature. With the growing utilization of these devices in modern dentistry, a certain number of implants may fail at the early stages of remodeling due to bacterial contamination. ${ }^{2}$ Accordingly, this situation increased the demand for antibacterial coverage to reduce the chances of failure. Thus, it may be argued that preoperative prescription of antibiotics to reduce any potential infections or implant failures is advocated especially when local and/or systemic factors are present. ${ }^{3,4}$

The beneficial role of antibiotics had conflicted results in the literature. Laskin et al. reported that high survival rate of implants was found in patients who received preoperative antibiotics. ${ }^{4}$ Alternatively, Gynther et al. reported that antibiotic administration had no effect in reducing postoperative complication and/or failures. ${ }^{5}$ With the controversies in the literature, a recent metaanalysis of randomized clinical trials showed that prophylactic antibiotics administration for low-risk, healthy patients may reduce the potential of implant failure. ${ }^{6}$ However, there were no conclusive findings about what specific antibiotic, dosage,
Periodontics Division, Department of Periodontics and Community Dentistry, College of Dentistry, King Saud University, Riyadh, Kingdom of Saudi Arabia

Corresponding Author: Mohammed A AlSarhan, Periodontics Division, Department of Periodontics and Community Dentistry, College of Dentistry, King Saud University, Riyadh, Kingdom of Saudi Arabia, Phone: +966 505556442, e-mail: malsarhan@ksu.edu.sa

How to cite this article: AlSarhan MA. Knowledge and Prescription Habits Toward Preoperative Antibiotics in Implant Dentistry: A Survey Analysis in a Subset of Dentists in Saudi Arabia. J Contemp Dent Pract 2020;21(5):568-574.

Source of support: Nil

Conflict of interest: None

duration, and any confounding variables that may have influenced the outcomes.

Globally, the potential risk of prescribing antibiotics for low-risk dental implant patients arise from the recent reports of bacterialresistant species that may complicate the future general health of different human populations. ${ }^{7}$ In addition, the required knowledge of different types of antibiotics is crucial for dentists who practice implant dentistry on regular bases. ${ }^{8}$ Although growing evidence 
from the literature suggest that preoperative administration of antibiotics may decrease the event of failures with minimal postoperative complications, ${ }^{9,10}$ it is of utmost importance for dentist to understand and implement prescription habits that have an acceptable level of balance between potential risks and benefits according to patients individual needs. ${ }^{11}$

Several reports describe the knowledge, habits, and behaviors regarding prescription of antibiotics among populations of healthcare providers. In dentistry, the most common themes for prescribing antibiotic medications were evident in conditions related to infected teeth and dental implants. ${ }^{12}$ However, a distinctive variation toward type, dose, and frequency for antibiotic administration was noticed. ${ }^{13,14}$ Addressing this issue as early as possible in dentist careers is crucial to establish drug management policies and provide medication prescription stewardship programs in dental education. Thus, our primary objective is to investigate the knowledge and practice toward preoperative antibiotics prescription in healthy dental implant patients among dentists in Saudi Arabia. Second, we want to explore and identify prescription habits toward specific clinical and nonclinical situations among sample participants.

\section{Materials and Methods}

After receiving institution review approval (no. E-19-4278), a subset of dental practitioners was targeted using the database of the Saudi commission for health specialties. An online-based survey was distributed to licensed dental practitioners, of different specialties and education levels, in central region of Saudi Arabia. The survey construction was formulated after reviewing recent publications of the subject in matter. ${ }^{6,14}$ About 200 potential participants who currently practice the surgical placement of dental implants were approached to participate. Only those who did not completed the whole survey were excluded from the study sample. The survey questionnaires consisted of 24 items; 8 questions covered the basic demographics of participant. Then, the survey was prepared to address the research objectives in three parts: the first part consisted of six items that targeted the knowledge level of participants toward antibiotics use in implant dentistry (Table 2). The second part involved seven items evaluating the habits and practice of preoperative antibiotics prescription in routine dental implant procedures (Table 3). Finally, three items addressed participants' background of acquired knowledge in addition to certain habits of prescribing preoperative antibiotics toward specific case scenarios (Table 5). The initial draft of the survey questionnaire was reviewed by two independent specialists for consistency, followed by reliability and validity testing and retesting on five postgraduates and five specialists within 1-week period. The final version was then distributed via web-based forms (Google Forms; Google LLC, Mountain View, CA, USA) and response was recorded and tabulated. Data were analyzed using SPSS 24.0 version (IBM Inc., Chicago USA) statistical software. Descriptive statistics (frequencies and percentages) were used to describe the categorical variables. Pearson's Chi-square test was used to compare the distribution of the categorical responses across focused survey variables. A $p$ value of $\leq 0.05$ was used to report the statistical significance of the responses.

\section{Results}

Only 156 completed the whole survey with a response rate of $78 \%$ from the original participants. A total of 120 (76.9\%) participants were male subjects. Only $21.2 \%$ of them were with bachelor's degree, and the remaining participants were with higher educational qualification. There was approximately a comparable distribution of dental residents and practitioners among the sample study with more than two third of them were periodontics specialty. The experience in surgical implants placement was about $48.1 \%$ of them having less than 5 years of practice, while the place of practice was distributed equally across academics, public, and private sectors. One participant did not respond to the question "source of dental education" by leaving a blank statement and was excluded from the analysis for this item. Thus, of 155 participants, more than $60 \%$ of them had postgraduate residency as their source of dental implant education followed by implant fellowship programs (23.9\%). A summary of sample characteristics is shown in Table 1.

\section{Knowledge of Antibiotics Prescription}

The assessment of knowledge of antibiotics prescription was carried out using six questions where responses were recorded as binary and 3-point nominal scales. About 63.5\% $(n=99)$ of the study subjects do not prescribe antibiotics preoperatively in routine dental practice. A total of $44.9 \%(n=70)$ reported no difference in therapeutic effect between single- and multidose prescription and responded positively aware about clinical guidelines or

Table 1: Characteristics and educational profile of study subjects $(n=156)$

\begin{tabular}{lc}
\hline Characteristics & No. $(\%)$ \\
\hline Gender & \\
Female & $36(23.1)$ \\
Male & $120(76.9)$ \\
Educational qualification & \\
Bachelor's degree & $33(21.2)$ \\
Master's degree & $48(30.8)$ \\
Board certified & $19(12.2)$ \\
PhD & $56(35.9)$ \\
Level of specialization & \\
Dental resident & $75(48.1)$ \\
Dental practitioner & $81(51.9)$ \\
Clinical specialty & \\
General practitioner & $14(9)$ \\
Oral and maxillofacial surgery & $25(16)$ \\
Periodontist & $110(70.5)$ \\
Prosthodontist & $7(4.5)$ \\
Years of practice in dental implant surgery & \\
Less than 5 years & $75(48.1)$ \\
5-10 years & $53(34)$ \\
10-20 years & $17(10.9)$ \\
More than 20 years & $11(7.1)$ \\
Place of practice & \\
Academic & $52(33.3)$ \\
Public & $51(32.7)$ \\
Private & $53(34)$ \\
Postgraduate residency & \\
Continuous dental education & $23(14.8)$ \\
\hline
\end{tabular}


Table 2: Distribution of responses toward knowledge of antibiotics prescription in dental implant surgery $(n=156)$

\begin{tabular}{|c|c|}
\hline Items of knowledge and practice & No. (\%) \\
\hline \multicolumn{2}{|c|}{$\begin{array}{l}\text { Would you prescribe preoperative antibiotics for patients receiving } \\
\text { routine dental implants? }\end{array}$} \\
\hline Yes & $57(36.5)$ \\
\hline No & $99(63.5)$ \\
\hline \multicolumn{2}{|c|}{$\begin{array}{l}\text { In your opinion, a single dose has the same therapeutic effect as } \\
\text { multidose of preoperative antibiotics for routine dental implant } \\
\text { surgery }\end{array}$} \\
\hline Yes & $70(44.9)$ \\
\hline No & $45(28.8)$ \\
\hline I am not sure & $41(26.3)$ \\
\hline \multicolumn{2}{|c|}{$\begin{array}{l}\text { Are you aware of clinical guidelines or consensus about } \\
\text { preoperative antibiotic prescription in routine dental implant } \\
\text { placement? }\end{array}$} \\
\hline Yes & $110(70.5)$ \\
\hline No & $46(29.5)$ \\
\hline \multicolumn{2}{|c|}{$\begin{array}{l}\text { Do you think there is a need for established national/international } \\
\text { guidelines on antibiotic prescription with dental implant therapy? }\end{array}$} \\
\hline Yes & $133(85.3)$ \\
\hline No & $23(14.7)$ \\
\hline \multicolumn{2}{|c|}{$\begin{array}{l}\text { Prescribing a single dose of preoperative antibiotics may lead to } \\
\text { unwanted adverse events or drug interactions? }\end{array}$} \\
\hline Yes & $56(35.9)$ \\
\hline No & $66(42.3)$ \\
\hline I am not sure & $34(21.8)$ \\
\hline \multicolumn{2}{|c|}{$\begin{array}{l}\text { Prescribing a multidose of preoperative antibiotics may lead to } \\
\text { unwanted adverse events or drug interactions? }\end{array}$} \\
\hline Yes & $76(48.7)$ \\
\hline No & $44(28.2)$ \\
\hline I am not sure & $36(23.1)$ \\
\hline
\end{tabular}

consensus regarding preoperative antibiotic prescription $(70.5 \%$, $n=110$ ). For the need to establish national/international guidelines on antibiotic prescription with dental implant therapy, a higher proportion of subjects $(85.3 \%, n=133)$ responded positively. Interestingly, only $35.9 \%$ and $48.7 \%$ agreed that adverse events may arise when prescribing a single and multiple dose of prophylactic antibiotics, respectively. More details of respondent's frequency and percentage are reported in Table 2.

\section{Practice of Antibiotics Prescription}

The assessment of practice of antibiotics prescription was carried out using six questions where responses were observed on ordinal and nominal scales. A total of $64.1 \%(n=100)$ of participants stated that they "always" explain to their patient the potential risk vs benefits of prescribed antibiotics. A greater tendency $(67.9 \%, n=106)$ of the collected sample would start their patient on antibiotics 1 hour preoperatively, with elevated responses $(61.5 \%, n=96)$ who prefer prescribing amoxicillin and augmentin, while considering a dose of 1000 and $2000 \mathrm{mg}$ in a single-dose regimen $(77.6 \%, n=121)$, whereas most of the sample $(96.2 \%, n=$ 150) agreed to choose twice and three times daily for a multidose regimen. Finally, more than two thirds of the participants found no preference in location of implant placement and prescription habits (Table 3 ).
Table 5: Distribution of responses toward the sources of knowledge and attitudes of prescription of antibiotics during dental implant surgery $(n=156)$

\begin{tabular}{lc}
\hline Items of knowledge and attitudes* & No. (\%) \\
\hline Which of the following primary sources increased your knowledge \\
and practice of prescribing a preoperative antibiotic? \\
Previous training (postgraduate training) & $101(64.7)$ \\
Scientific journals, local literature, textbook & $106(67.9)$ \\
Continuing education courses or seminars & $77(49.4)$ \\
Internet & $24(15.4)$ \\
Guidelines from professional organizations & $65(41.7)$ \\
Informal contact with colleagues or verbal & $27(17.3)$ \\
communication & \\
Personal experience or self-directed learning & $52(33.3)$ \\
Advertisement or drug sale representatives & $5(3.2)$ \\
In our opinion, the primary goal for prescribing preoperative \\
antibiotics in routine dental implant patients is \\
Favorable wound healing and osseointegration \\
Decrease chance of implant failure & $31(19.9)$ \\
Reduce site contamination and infection & $52(33.3)$ \\
Other & $133(85.3)$
\end{tabular}

Which of the following conditions will you prescribe preoperative antibiotics in routine dental implant patients?

Elderly patients

$43(27.9)$

Thin gingival biotype

$15(9.7)$

History of periodontal disease

$66(42.9)$

History of failed implants/periimplantitis

$76(49.4)$

Multiple implant placement

$58(37.7)$

Suboptimal plaque control

$18(11.7)$

Long surgical procedures

61 (39.6)

Smokers

50 (32.5)

Controlled diabetes

45 (29.2)

Prosthetic joint replacement

$74(48.1)$

Congenital heart disease

$92(59.7)$

Infective endocarditis

$128(83.1)$

Recent myocardial infarction

$83(53.9)$

Coronary artery bypass surgery

75 (48.7)

Mitral valve prolapses

$80(51.9)$

Rheumatic heart disease

$93(60.4)$

No specific reason

$10(6.5)$

*Multiple responses

\section{Knowledge and Practice of Antibiotics Prescription Responses in Relation to the Working Sector}

An additional analysis comparing responses toward knowledge and practice of antibiotics prescription among the three working sectors (academic, public, and private) shows a statistically significant difference for the following three questions: (i) Would you prescribe preoperative antibiotics for patients receiving routine dental implants? (ii) Do you think there is a need for established national/international guidelines on antibiotic prescription with dental implant therapy? and (iii) Prescribing a multidose of preoperative antibiotics may lead to unwanted adverse events or drug interactions? Dentist who practice in the private sector $(47.4 \%, n=27)$ found to prescribe more antibiotics 
Table 3: Distribution of responses toward practice of antibiotics prescription in dental implant surgery $(n=156)$

\begin{tabular}{lr}
\hline Items of practice & No. (\%) \\
\hline \multicolumn{2}{l}{ Do you explain to your patients the potential risk vs benefits of } \\
prescribed antibiotics? & $100(64.1)$ \\
$\quad$ Always & $37(23.7)$ \\
Sometimes & $19(12.2)$ \\
$\quad$ Never & \\
When prescribing preoperative antibiotics, when would you start \\
your patient on? \\
Immediately before implant placement \\
30 minutes preoperatively & $13(8.8)$ \\
1 hour preoperatively & $106(67.9)$ \\
2 hours preoperatively & $23(14.7)$ \\
Other & $8(5.1)$ \\
In routine and healthy dental implant patients, what type of \\
preoperative antibiotic would you prescribe? \\
Amoxicillin & $96(61.5)$ \\
Augmentin & $54(34.6)$ \\
Clindamycin & $5(3.2)$ \\
Cephalexin & $1(0.6)$
\end{tabular}

Based on your previous selection, what dosage of preoperative antibiotic would you choose?

$\begin{array}{lc}150 \mathrm{mg} & 2(1.3) \\ 300 \mathrm{mg} & 2(1.3) \\ 500 \mathrm{mg} & 24(15.4) \\ 875 \mathrm{mg} & 1(0.6) \\ 1000 \mathrm{mg} & 63(40.4) \\ 2000 \mathrm{mg} & 58(37.2) \\ 3000 \mathrm{mg} & 3(1.9) \\ \text { Other } & 3(1.9)\end{array}$

For a multidose regimen, which of the following will you prescribe?

$\begin{array}{lc}\text { BID (twice a day) } & 68(43.6) \\ \text { TID (three times a day) } & 82(52.6) \\ \text { QID (four times a day) } & 3(1.9) \\ \text { Other } & 3(1.9)\end{array}$

In your opinion, which anatomical locations of jaws will most likely need preoperative antibiotics?

$\begin{array}{lc}\text { Upper anterior region } & 3(1.9) \\ \text { Upper posterior region } & 23(14.7) \\ \text { Lower anterior region } & 7(4.5) \\ \text { Lower posterior region } & 11(7.1) \\ \text { No specific region } & 112(71.8)\end{array}$

preoperatively, while in academic sector $(39.4 \%, n=39)$ was not in favor of prescription which is statistically significant $(p=0.019)$. Interestingly, an increased percentage of private practitioners responded negatively to the need for established guidelines in prescribing antibiotics $(60.9 \%, n=14)$, and this was highly significant ( $p=0.009$ ). For the question (iii), 32.1 and $37.5 \%$ of study subjects who were working in academic and public sectors had responded with "Yes," whereas $54.5 \%$ who were in private sector had responded as "No," which was statistically significant $(p=0.014)$. A further analysis shows no statistically significant difference in the responses of subjects for the remaining four questions (Table 4).

\section{Sources of Knowledge and Attitudes of Prescription}

The sources of knowledge and attitudes of prescribing prophylactic antibiotics during routine dental implant surgery were assessed by multiple responses, with all possible options. More than $50 \%$ of the sample had stated that previous training (postgraduate training), scientific journals, local literature, and textbooks as their main source of knowledge, while $49.4 \%$ were of the view to gain it through continuing education courses or seminars. While other unpopular sources of knowledge were found to be the internet (15.4\%), informal contact with colleagues or verbal communication (17.3\%), and personal experience or drug sale representatives (33.3 and 3.2\%, respectively). A higher proportion of respondents (85.3\%) believe that preoperative prescription is useful in reducing site contamination and infection. And while majority of study sample would prescribe prophylactic antibiotics for systematic conditions like infective endocarditis (83.1\%) and rheumatic heart disease (60.4\%). Others tend to apply this habit for conditions with history of periodontal disease (42.9\%), history of failed implants/ periimplantitis (49.4\%), prosthetic joint replacement (48.1\%), recent myocardial infarction (53.9\%), coronary artery bypass surgery (48.7\%), mitral valve prolapse (51.9\%), and smokers (32.5\%). A detailed summary of respondent's percentage with other factors can be shown in Table 5 .

\section{Discussion}

Our findings showed that an increased number of participants do not prescribe prophylactic antibiotics in routine dental implant surgery (63.5\%). This is conflicting with recently reported similar studies by Al-Kattan, Al-Shibani and El-Kholey et al. where both reports had higher percentage of prescribing habits preoperatively. ${ }^{14-16}$ A possible explanation of this might be the lack of adequate sample size between the two investigations and the current work. Also, the variability of sample characteristics might suggest why responses where different, where in this study we included periodontist for both training residents and specialist. Froum and Weinberg reported that periodontists' prescription was variable for prophylactic antibiotics, and less than $35 \%$ would choose to administer preoperatively. ${ }^{17}$ This agrees with our findings especially that most of the respondents were periodontists of both groups. Another possible factor that may contribute to our findings is the increased awareness and strict measurements that direct dental practitioner to limit the use of antibiotics for low-risk patients and was evident in the decline of prophylactic antibiotics for routine implant procedures. ${ }^{18}$

In this study, we found that an elevated number of participants were aware of guidelines and/or consensus pertaining to prophylactic antibiotics which is similar to previous reports. ${ }^{14}$ While most of the respondents suggest the need to establish protocols in this matter, it was found that private practitioners were not in agreement with this issue. In addition, the significance of private practitioners to prescribe more antibiotics in comparison to other working sectors was evident (Table 2). This may be explained by the difference in workplace protocols where non-private sectors mandate strict policies and procedures for antibiotic prescriptions. Erbay et al. found that restrictive policies for antibiotics may aid in the reduction of unnecessary prescription habits. ${ }^{18}$ Furthermore, the interventional policies was found to have a positive impact in behavior change among dental practitioners and support the reduction of antibiotic prescription misuse. ${ }^{19}$ In a consensus report by the European Association of Osseointegration, one of 
Table 4: Comparison of responses toward knowledge and practice of antibiotics prescription in dental implant surgery in relation to working sector of dental implant practice of study subjects

\begin{tabular}{|c|c|c|c|c|c|}
\hline \multirow{2}{*}{$\begin{array}{l}\text { Items of knowledge and } \\
\text { practice }\end{array}$} & \multicolumn{3}{|c|}{ Working sector of dental implant practice } & \multirow[b]{2}{*}{$\chi^{2}$ value } & \multirow[b]{2}{*}{ pvalue } \\
\hline & Academic & Public & Private & & \\
\hline \multicolumn{6}{|c|}{ Would you prescribe preoperative antibiotics for patients receiving routine dental implants? } \\
\hline Yes & $13(22.8)$ & $17(29.8)$ & $27(47.4)$ & 7.954 & $0.019^{*}$ \\
\hline No & $39(39.4)$ & $34(34.3)$ & $26(26.3$ & & \\
\hline \multicolumn{6}{|c|}{ In your opinion, a single dose has the same therapeutic effect as multidose of preoperative antibiotics for routine dental implant surger } \\
\hline Yes & $25(35.7)$ & $22(31.4)$ & $23(32.9)$ & 7.53 & 0.11 \\
\hline No & $11(24.4)$ & $21(46.7)$ & $13(28.9)$ & & \\
\hline Not sure & $16(39)$ & $8(19.5)$ & $17(41.5)$ & & \\
\hline \multicolumn{6}{|c|}{ Are you aware of clinical guidelines or consensus about preoperative antibiotic prescription in routine dental implant placement? } \\
\hline Yes & $32(29.1)$ & $39(35.5)$ & $39(35.5)$ & 3.125 & 0.21 \\
\hline No & $20(43.5)$ & $12(26.1)$ & $14(30.4)$ & & \\
\hline \multicolumn{6}{|c|}{ Do you think there is a need for established national/international guidelines on antibiotic prescription with dental implant therapy? } \\
\hline Yes & $49(36.9)$ & $45(33.8)$ & $39(29.3)$ & 9.436 & $0.009^{* *}$ \\
\hline No & $3(13)$ & $6(26.1)$ & $14(60.9)$ & & \\
\hline \multicolumn{6}{|c|}{ Prescribing a single dose of preoperative antibiotics may lead to unwanted adverse events or drug interactions? } \\
\hline Yes & $18(32.1)$ & $21(37.5)$ & $17(30.4)$ & 1.306 & 0.86 \\
\hline No & $22(33.3)$ & $19(28.8)$ & $25(37.9)$ & & \\
\hline Not sure & $12(35.3)$ & $11(32.4)$ & $11(32.4)$ & & \\
\hline \multicolumn{6}{|c|}{ Prescribing a multidose of preoperative antibiotics may lead to unwanted adverse events or drug interactions? } \\
\hline Yes & $28(36.8)$ & $30(39.5)$ & $18(23.7)$ & 12.528 & $0.014^{*}$ \\
\hline No & $10(22.7)$ & $10(22.7)$ & $24(54.5)$ & & \\
\hline Not sure & $14(38.9)$ & $11(30.6)$ & $11(30.6)$ & & \\
\hline \multicolumn{6}{|c|}{ How often do you prescribe antibiotics preoperative for your patients in routine dental implant surgery? } \\
\hline Always & $11(26.8)$ & $10(24.4)$ & $20(48.8)$ & 8.097 & 0.088 \\
\hline Sometimes & $11(27.5)$ & $14(35)$ & $15(37.5)$ & & \\
\hline Never & $30(40)$ & $27(36)$ & $18(24)$ & & \\
\hline \multicolumn{6}{|c|}{ Do you explain to your patients the potential risk vs benefits of prescribed antibiotics? } \\
\hline Always & $35(35)$ & $28(28)$ & $37(37)$ & 7.314 & 0.12 \\
\hline Sometimes & $8(21.6)$ & $18(48.6)$ & $11(29.7)$ & & \\
\hline Never & $9(47.4)$ & $5(26.3)$ & $5(26.3)$ & & \\
\hline
\end{tabular}

*Statistically significant $p<0.05,{ }^{* *}$ statistically significant $p<0.01$

the main conclusions found that there is no beneficial impact of antibiotic prophylaxis for straightforward dental implant scenarios. ${ }^{20}$ Furthermore, the recent meta-analysis done by Braun et al. supports this conclusion. ${ }^{6}$ Accordingly, it is advocated that policies and interventions should be expanded to involve private practitioners too.

The balance between risk and benefit when prescribing prophylactic antibiotics is a crucial step for the overall management in dental implantology. Additionally, choosing between single and multiple prophylactic antibiotics has been reported to be of variable importance. El-Kholey, in a pilot study, found that there is no difference between both modalities of prescriptions regarding implant survival. ${ }^{21}$ One of the highlights of this study is the increased level of knowledge toward the possible unwanted adverse effects with the use of multidose compared with single-dose prophylactic antibiotics. This is supported by recent studies where increased adverse events were more evident in multidose regimens. ${ }^{9,10}$ While it is argued that a highly concentrated antibiotic regimen will provide similar therapeutic outcomes to a multiple dose, but with minimal adverse events in routine implant placement, ${ }^{10}$ the importance of consenting the potential risk vs benefits and tailoring decision making with prophylactic antibiotics in accordance with patients' needs could help in minimizing prescription overdosing and promote a better prescription practice. Although we did not investigate the method of consenting, most of the respondents shared a positive agreement in this matter (Table 4).

Most of the respondents in this study preferred the 1-hour protocol preoperatively, with amoxicillin/augmentin group and a dose of 1000-2000 mg. Along the way, twice and three times daily was the preferred choice for a multidose prescription. This agrees with previous reports done by Al-Kattan and Al-Shibani, where most participants prescribe $1000 \mathrm{mg}$ of amoxicillin. ${ }^{14}$ Also, our respondents were similar in the matter of choosing either amoxicillin/augmentin preoperatively and were similar to results obtained by El-Kholey et al. ${ }^{22}$ However, their sample study tends to prescribe more postoperative medication compared with the present investigation (Table 3). Esposito et al. suggested that $2 \mathrm{~g}$ of amoxicillin administered 1 hour before the implant procedure led to the reduction of implant infection and failure. ${ }^{23}$ On the contrary, Park et al. reported that there is no clinical advantage of antibiotic 
prophylaxis for routine patients receiving dental implants. ${ }^{24}$ However, in a recent metal-analysis of randomized clinical trials, Braun et al. found that prescription pattern of either preoperatively and/or postoperatively was significantly different when compared with no prescription and/or placebo. ${ }^{6}$ Although the meta-analysis was concerned with the implant infection/failure as the primary outcome, it is worth mentioning that lowest failure rate was best achieved for the prophylactic prescriptions. ${ }^{6}$ Thus, variable reports among national ${ }^{14,22}$ and international ${ }^{13,25}$ toward prescription patterns need to addressed by local and global professional and scientific organizations to provide more collaborative work to ultimately educate dental practitioners and trainees about most recent consensus and available evidence.

One of the important aspects of this study is that we tried to explore participants knowledge and habits with the practice of antibiotic prescriptions when challenged by local, environmental, and systemic diseases/conditions in addition to the background of information they acquired. Majority of study samples relied on previous training programs and scientific publications as the primary source of knowledge. This finding was not reported in previous studies. Furthermore, the selected sample reported that the main purpose of antibiotic prescription was to reduce infection of dental implants (Table 5). This can be explained by the fact that dental implant surgery is an invasive procedure which may increase the chance of developing infections and thus requires chemotherapeutic coverage to reduce the chance of bacterial contamination. ${ }^{26}$ Also, we identified that a moderate percentage of participants prescribe antibiotics for elderly patients or with systematic conditions including rheumatic heart disease, coronary artery bypass graft, mitral valve prolapse, and prosthetic joint replacement $(60.4,48.7,51.9$, and $48.1 \%$, respectively). However, recent consensus does not support prophylactic antibiotics in these situations anymore. ${ }^{27,28}$ These findings suggest that traditional ways of acquiring knowledge may need to be revised. Nonetheless, a growing evidence is advocating the necessity of practice stewardship directed to dental practitioners to avoid misuse and needless antibiotic prescriptions.

Despite its exploratory nature of this study, we attempted to address several factors pertaining to the knowledge and behavior of preoperative antibiotics. Also, we carried out further investigations to analyze clinical and nonclinical factors that influence prescription behaviors. We understand that the sample size achieved in our study need to be larger and involve other regions within Saudi Arabia. However, with the extensive variables acquired through the survey items and focusing on one region of the country, we successfully identified a significant factor which was not previously reported and requires more attention. Also, further studies which take these variables into account will need to be undertaken to develop a full picture of this topic. Furthermore, with the diversity of responses and contradictory results regarding similar studies entails the need to have a more revision and contemplation of what we considered before as "reputable" source of knowledge starting from dental schools. Finally, the implementation of recent guidelines and administrative supervision in all dental sectors should be considered by health-care officials.

\section{Conclusion}

Despite the study limitations, this work offers valuable insights into knowledge and certain habits of dental implant practitioners toward prophylactic antibiotics. The methods used for this survey may be applied to large-scale analysis to have better understanding of different behaviors and beliefs. Also, the presented diversity and conflicting opinions require more attention by health authorities to develop a standard guideline that is backed up with recent evidence-based research to reduce nonessential antibiotic prescription.

\section{References}

1. McGuire MK, Wilson Jr TG. Commentary: from normal scientific progress to game changers: the impact on periodontal clinical practice. J Periodontol 2014;85(8):1001-1005. DOI: 10.1902/ jop.2014.140220.

2. Sakka S, Baroudi K, Nassani MZ. Factors associated with early and late failure of dental implants. J Investig Clin Dent 2012;3(4):258-261. DOI: 10.1111/j.2041-1626.2012.00162.x.

3. Marchand F, Raskin A, Dionnes-Hornes A, et al. Dental implants and diabetes: conditions for success. Diabetes Metab 2012;38(1):14-19. DOI: 10.1016/j.diabet.2011.10.002.

4. Laskin DM, Dent CD, Morris HF, et al. The influence of preoperative antibiotics on success of endosseous implants at 36 months. Ann Periodontol 2000;5(1):166-174. DOI: 10.1902/annals.2000.5.1.166.

5. Gynther GW, Köndell PÅ, Moberg L-E, et al. Dental implant installation without antibiotic prophylaxis. Oral Surg Oral Med Oral Pathol Oral Radiol Endod 1998;85(5):509-511. DOI: 10.1016/S1079-2104(98) 90281-5.

6. Braun RS, Chambrone L, Khouly I. Prophylactic antibiotic regimens in dental implant failure: a systematic review and meta-analysis. J Am Dent Assoc 2019;150(6):e61-e91. DOI: 10.1016/j.adaj.2018.10.015.

7. Sweeney LC, Dave J, Chambers PA, et al. Antibiotic resistance in general dental practice-a cause for concern? J Antimicrob Chemother 2004;53(4):567-576. DOI: 10.1093/jac/dkh137.

8. Roberts MC. Antibiotic toxicity, interactions and resistance development. Periodontol 2000 2002;28(1):280-297. DOI: 10.1034/j.1600-0757.2002.280112.x.

9. Binahmed A, Stoykewych A, Peterson L. Single preoperative dose versus long-term prophylactic antibiotic regimens in dental implant surgery. Int J Oral Maxillofac Implants 2005;20(1):115-117.

10. Arduino PG, Tirone F, Schiorlin E, et al. Single preoperative dose of prophylactic amoxicillin versus a 2 -day postoperative course in dental implant surgery: a two-centre randomised controlled trial. Eur J Oral Implantol 2015;8(2):143-149.

11. Aronson JK. Balanced prescribing - principles and challenges. Br J Clin Pharmacol 2012;74(4):566-572. DOI: 10.1111/j.1365-2125.2012.04413.x.

12. Marra F, George $D$, Chong $M$, et al. Antibiotic prescribing by dentists has increased: why? J Am Dent Assoc 2016;147(5):320-327. DOI: 10.1016/j.adaj.2015.12.014.

13. Deeb GR, Soung GY, Best AM, et al. Antibiotic prescribing habits of oral and maxillofacial surgeons in conjunction with routine dental implant placement. J Oral Maxillofac Surg 2015;73(10):1926-1931. DOI: 10.1016/j.joms.2015.05.024.

14. Al-Kattan R, Al-Shibani N. Current trends in antibiotic prescription behavior among Saudi dentists performing implant surgery: a crosssectional observational study. J Investig Clin Dent 2019;10(2):e12383. DOI: 10.1111/jicd.12383.

15. El-Kholey KE, Wali O, Elkomy A, et al. Pattern of antibiotic prescription for oral implant treatment among dentists in Saudi Arabia. Implant dentistry 2018;27(3):317-323.

16. Khalil $D$, Hultin $M$, Andersson Fred $L$, et al. Antibiotic prescription patterns among Swedish dentists working with dental implant surgery: adherence to recommendations. Clin Oral Implants Res 2015;26(9):1064-1069. DOI: 10.1111/clr.12402.

17. Froum SJ, Weinberg MA. An evaluation of antibiotic use in periodontal and implant practices. Int J Periodontics Restorative Dent 2015;35(4):481-487. DOI: 10.11607/prd.2488.

18. Erbay A, Çolpan A, Bodur $\mathrm{H}$, et al. Evaluation of antibiotic use in a hospital with an antibiotic restriction policy. Int J Antimicrob Agents 2003;21(4):308-312. DOI: 10.1016/S0924-8579(02)00392-8. 
19. Kim H, Oh JK, Kim MK, et al. Reduced antibiotic prescription rates following physician-targeted interventions in a dental practice. Acta Odontol Scand 2018;76(3):204-211. DOI: 10.1080/ 00016357.2017.1402209.

20. Klinge B, Flemming T, Cosyn J, et al. The patient undergoing implant therapy. Summary and consensus statements. The 4th EAO Consensus Conference 2015. Clin Oral Implants Res 2015;26(Suppl 11): 64-67. DOI: 10.1111/clr.12675.

21. El-Kholey K. Efficacy of two antibiotic regimens in the reduction of early dental implant failure: a pilot study. Int J Oral Maxillofac Surg 2014;43(4):487-490. DOI: 10.1016/j.ijom.2013.09.013.

22. El-Kholey KE, Wali O, Elkomy A, et al. Pattern of antibiotic prescription for oral implant treatment among dentists in Saudi Arabia. Implant Dent 2018;27(3):317-323. DOI: 10.1097/ID.0000000000000748.

23. Esposito M, Cannizzaro G, Bozzoli P, et al. Effectiveness of prophylactic antibiotics at placement of dental implants: a pragmatic multicentre placebo-controlled randomised clinical trial. Eur J Oral Implantol 2010;3(2):135-143.

24. Park J, Tennant $M$, Walsh $L J$, et al. Is there a consensus on antibiotic usage for dental implant placement in healthy patients? Aust Dent J 2018;63(1):25-33. DOI: 10.1111/adj.12535.
25. Datta R, Grewal Y, Batth J, et al. Current trend of antimicrobial prescription for oral implant surgery among dentists in India. J Maxillofac Oral Surg 2014;13(4):503-507. DOI: 10.1007/s12663-0130567-7.

26. Pye $A$, Lockhart $D$, Dawson $M$, et al. A review of dental implants and infection. J Hosp Infect 2009;72(2):104-110. DOI: 10.1016/ j.jhin.2009.02.010.

27. Wilson W, Taubert KA, Gewitz M, et al. Prevention of infective endocarditis: guidelines from the American Heart Association: a guideline from the American Heart Association Rheumatic Fever, Endocarditis, and Kawasaki Disease Committee, Council on Cardiovascular Disease in the Young, and the Council on Clinical Cardiology, Council on Cardiovascular Surgery and Anesthesia, and the quality of care and outcomes research interdisciplinary working group. Circulation 2007;116(15):1736-1754. DOI: 10.1161/CIRCULATIONAHA. 106.183095.

28. Sollecito TP, Abt E, Lockhart PB, et al. The use of prophylactic antibiotics prior to dental procedures in patients with prosthetic joints: evidencebased clinical practice guideline for dental practitioners-a report of the American Dental Association Council on Scientific Affairs. J Am Dent Assoc 2015;146(1):11.e8-16.e8. DOI: 10.1016/j.adaj.2014. 11.012 . 